\title{
Formation of non-classical optical states in spaser systems under control of an external magnetic field
}

\author{
Mikhail Gubin and Alexei Prokhorov* \\ Vladimir State University named after A. G. and N. G. Stoletovs, 600000 Vladimir, Russia
}

\begin{abstract}
The work is focused on the investigation of features of quantum dynamics for photons in spaser systems consisting of metal nanoparticles (NP) and semiconductor quantum dots (QDs). The non-classical photon states generation in a three-particle spaser system with nonlinear plasmonexciton interaction is predicted.
\end{abstract}

Ensembles of near-field coupled nanoparticles (NPs) and quantum dots (QDs) are of significant interest as a platform for superresolution microscopy of nanostructures [1]. In fact, the bunching (antibunching) effects can be observed for emitted photons from self-assembled QD structures [2]. On the other hand, if QDs are coupled with shared NP [3], direct control of quantum statistics and correlation properties of generated photons in such spaser-like system can be realized. In particular, the control of generation of non-classical light in the described ensembles will allow to realize the spatial superresolution $[4,5]$ using simple and cheap nanoscale sensors.

In this paper we propose a model of three nanoobjects (NP-QD-NP) coupled by nonlinear dipole-dipole interactions in the presence of an external magnetic field, see Fig. 1a. The nonlinear regime of this ensemble corresponds to the two-quantum processes of the QD biexciton decay in the case $|\delta|>\Omega_{1,2},|\Delta|$, where $\delta=\varpi-\omega$ and $\Delta=\varpi-\omega_{p}, \varpi$ is the spasing frequency, and $\Omega_{1,2}$ are the Rabi frequencies of dipole-dipole interactions between the QD and NPs. In the technical framework, the presented system of radiative spaser can be used to generate nonclassical states of the electromagnetic field at the nanoscale. Nonlinear regimes of interaction between NP and QD can be realized, firstly, in the presence of a two-photon pump in the system and, secondly, under the condition that the coupling energy between two electron-hole pairs is of the same order of magnitude as the internal coupling energy of a single pair.

The system of Heisenberg-Langevin equations corresponding to nonlinear plasmonexciton interaction in the system can be represented in the following form [3]:

$$
\begin{gathered}
\dot{\hat{c}}_{1(2)}=i\left(\Delta+i / \tau_{c}\right) \hat{c}_{1(2)}-i \Omega^{(2)} \hat{c}_{2(1)}^{+} \hat{S}+\hat{F}_{c 1(c 2)}, \dot{\hat{S}}=i\left(\delta^{(2)}+i / \tau_{S}\right) \hat{S}+i \Omega^{(2)} \hat{c}_{1} \hat{c}_{2} D+\hat{F}_{S}, \\
\dot{D}=2 i \Omega^{(2)}\left(\hat{c}_{1}^{+} \hat{c}_{2}^{+} \hat{S}-\hat{S}^{+} \hat{c}_{1} \hat{c}_{2}\right)-\left(D-D_{0}\right) / \tau_{D}+\hat{F}_{D}
\end{gathered}
$$

\footnotetext{
* Corresponding author: avprokhorov33@mail.ru
} 

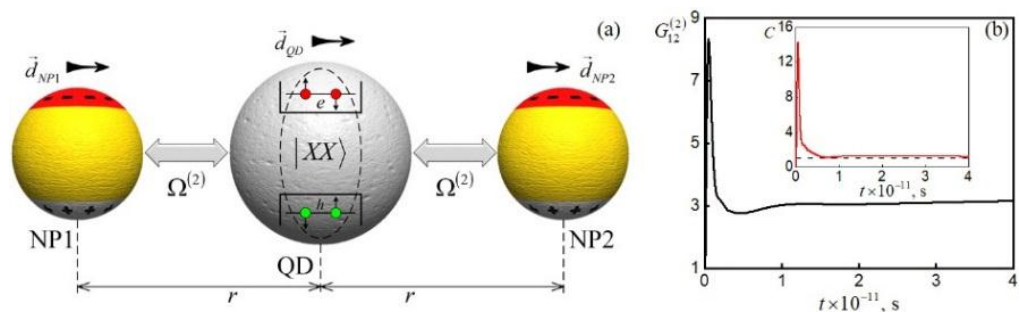

Fig. 1. (a) The model of a three-particle radiative NP-QD-NP spaser. (b) The time dependence of the cross-correlation $G_{12}^{(2)}$ and $C$ (inset) functions for photons generated in nonlinear NP-QD-NP spaser.

where $\hat{S}$ corresponds to the biexciton state of QD and the creation operators $\hat{c}_{1}^{+}$and $\hat{c}_{2}^{+}$ correspond to the pair of plasmons, whose energies slightly differ for the different intermediate levels with frequencies $\omega_{X_{+}}\left(\omega_{X_{-}}\right)$. The parameter $\Omega^{(2)}=\Omega_{1} \Omega_{2} /(2|\delta|)$ is the effective two-quantum Rabi frequency, $D$ is the population imbalance. Parameters $\delta=\varpi-\omega_{X}$ and $\delta^{(2)}=2 \varpi-\omega_{X X}$ are the effective detunings, where $\omega_{X X}=\omega_{X_{-}}+\omega_{X_{+}}-\Delta_{2 b}$ is the biexciton frequency; $\Delta=\varpi-\omega_{p}, \Delta_{2 b}$ is the biexciton binding energy. The Rabi frequency of interaction between the QD and NP is $\Omega_{1(2)}=\mu_{Q D} / r^{3} \sqrt{\omega_{p 1(2)} a_{N P 1(2)}^{3} /\left(2 \pi \varepsilon_{0} \hbar\right)}$, where $a_{N P}$ is the NP radius, $\omega_{p}$ is the plasmon resonance frequency of NPs, $\mu_{Q D}$ is the dipole moment. In this work, we analyse the dynamics of the cross-correlation function $G_{12}^{(2)}$ [3] for photons based on the dynamics of plasmons and excitons in the system [6], see Fig. 1b. In particular, the violation of the Cauchy-Schwarz inequality $C \equiv G_{12}^{(2)}(t, 0)^{2} /\left(g_{1}^{(2)}(t, 0) g_{2}^{(2)}(t, 0)\right) \leq 1$ (see Fig. 1b) indicates the nonclassical character of the correlations, where $g_{1(2)}^{(2)}=G_{1(2)}^{(2)}+1$. The maximum value of $C$ parameter is 14.24 and the stationary regime value is 1.2 , which demonstrates the nonclassical character of correlations between plasmon modes in spaser system. Thus, the main result of our simulation is the demonstration of the development of quantum correlations between modes and the possibility for the formation of entangled state of photons. Further development of this work may be aimed on a complex simulation of nonlinear processes with surface plasmon-polaritons generated in plasmonic circuits [7]. Special attention is required to study the temperature dependences of the discussed effects using real physical systems as an example [8].

\section{References}

1. I.Yu. Eremchev, M.Yu. Eremchev, A.V. Naumov, Phys. Usp. 62, 294 (2019)

2. C. Becher, A. Kiraz, P. Michler et al., Phys. Rev. B 63, 121312(R) (2001)

3. M.Yu. Gubin, A.V. Shesterikov, S.N. Karpov et al., Phys. Rev. B. 97, 085431 (2018)

4. A. Classen, J. von Zanthier, M.O. Scully et al., Optica 4, 580 (2017)

5. M.W. Mitchell, J.S. Lundeen, A.M. Steinberg, Nature 429, 161 (2004)

6. A.I. Fernández-Domínguez, S.I. Bozhevolnyi, N.A. Mortensen, ACS Photonics 5, 3447 (2018)

7. C. Reinhardt, A.B. Evlyukhin, W. Cheng et al., J. Opt. Soc. Am. B 30, 2898 (2013)

8. K.A. Magaryan, K.R. Karimullin, I.A. Vasil'eva et al., Opt. Spectrosc. 126, 41 (2019)

9. K.A. Magaryan, M.A. Mikhailov, K.R. Karimullin et al., J. Lumin. 169, 799 (2016) 